\title{
SEARCH FOR NEW PHYSICS WITH PHOTONS AT CDF
}

\author{
A. PRONKO \\ Fermilab, Batavia, IL, USA \\ E-mail: pronko@fnal.gov \\ (For the CDF Collaboration)
}

\begin{abstract}
We present searches for signals of new physics beyond the Standard Model. The focus of this paper is on the results obtained with photons in the final state. We report on two model-independent searches for anomalous production of $\gamma \gamma+X$ (where $X=e, \mu, \gamma, \mathbb{E}_{T}$ ) and $\gamma l+X$ (where $l=e, \mu$ and $X=l, \gamma, \mathbb{F}_{T}$ ) events and one novel search for delayed photons. The analyses are based on $\sim 1 \mathrm{fb}^{-1}$ of data and significantly update earlier results. No significant excess of data over the predicted background is observed. We report kinematic distributions, data and background counts.
\end{abstract}

\section{Model-independent Approach in Searches with Photons}

The single and di- photon signatures in the final state are present in many well motivated theoretical models of new physics beyond the Standard Model. Examples of such models include Gauge-Mediated SUSY (GMSB), mSUGRA, fermiophobic Higgs, RS-graviton production, LED, $b^{\prime}$ production, compositeness (exited fermions), etc. Chances that any particular model could be the one describing our world are very small. In light of this fact, CDF employed a model-independent approach in searching for new physics in inclusive $\gamma \gamma+X$ and $\gamma l+X$ final states. The analysis strategy for a model-independent search, unlike to conventional model-based searches, is not to optimize the selection requirements for any particular physics model, but rather to apply generic selection criteria to reduce backgrounds. An increased attention is also given to examining various kinematic distributions. Observation of an excess of data over the predicted background would indicate a presence of new physics.

\section{Search for Anomalous Production of $\gamma \gamma+X$ Events}

There were two very rare di-photon events recorded at the Tevatron experiments: Run

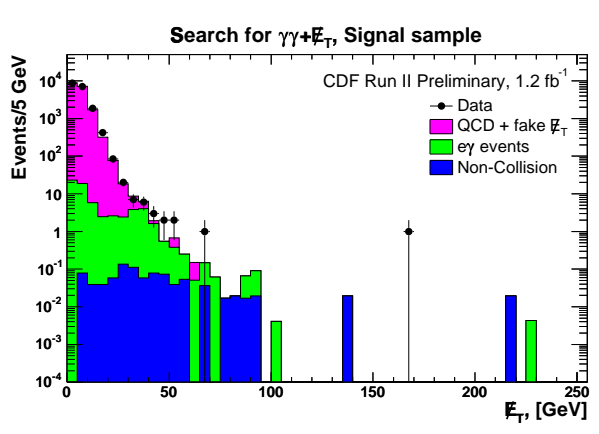

Fig. 1. Distribution of $\mathscr{F}_{T}$ in $\gamma \gamma$ events.

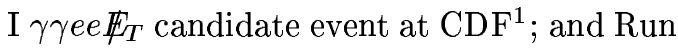
II $\gamma \gamma e \mathbb{F}_{T}$ event at $\mathrm{D}^{2}$. The observation of the $\gamma \gamma e e \mathbb{F}_{T}$ event is particularly interesting because the total predicted background is only $\sim 10^{-6}$ events, and this event could be a sign of new physics. With $\sim 12$ times more data in Run II, CDF has significantly higher potential for detecting signs of new physics in production of rare $\gamma \gamma+X$ events.

\subsection{The $\gamma \gamma+F_{T}$ Channel}

Figure 1 shows the $\mathbb{F}_{T}$ distribution in $\gamma \gamma$ events with two isolated photons with $E_{T}^{\gamma}>13 \mathrm{GeV}$ and located in the central region $\left(\left|\eta_{\gamma}\right|<1.1\right)$ of the calorimeter. The results are based on $\sim 1.2 \mathrm{fb}^{-1}$ of data. The overall agreement of data and the predicted background is good. At low values of $\mathbb{F}_{T}$, the 

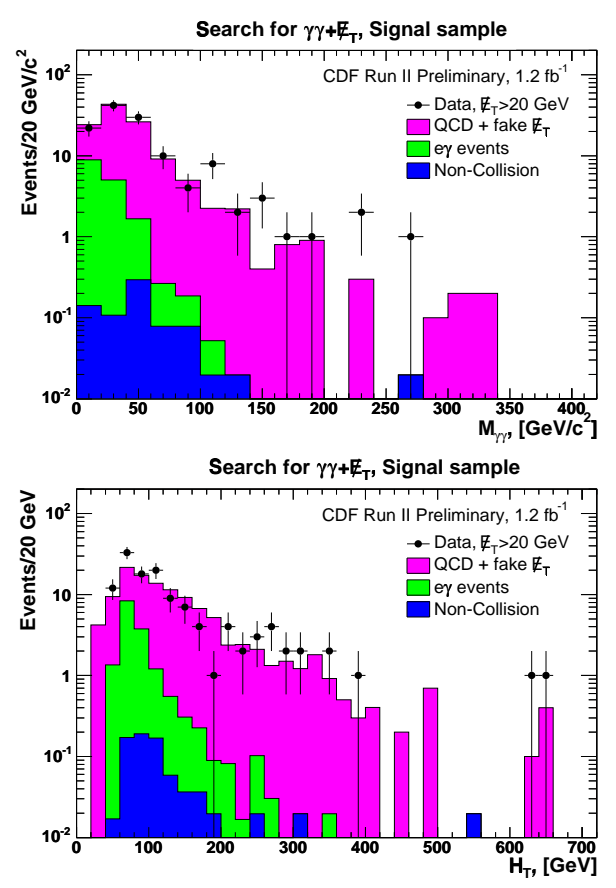

Fig. 2. The invariant mass, $M_{\gamma \gamma}(t o p)$, and $H_{T}$ (bottom) distributions in $\gamma \gamma$ events with $\mathbb{F}_{T}>20 \mathrm{GeV}$.

background is dominated by regular QCD events $^{\mathrm{a}}$ with fake ${ }^{\mathrm{b}} \mathbb{E}_{T}$. The region of intermediate values and tail of the distribution is mostly populated by $W \gamma \rightarrow e \bar{\nu} \gamma$ and noncollision $^{c}$ events where one or both photons are faked. We observe 22 events with $\mathbb{F}_{T}>30$ $\mathrm{GeV}$ at the expected background of $19.5 \pm 2.5$ events. We have also studied invariant mass, $M_{\gamma \gamma}$, of $\gamma \gamma$ pairs and $H_{T}{ }^{\mathrm{d}}$ distribution in the events with $\mathbb{E}_{T}>20 \mathrm{GeV}$ (Fig. 2). Tails of both $M_{\gamma \gamma}$ and $H_{T}$ histograms are dominated by QCD events with fake $\mathbb{F}_{T}$. No significant excess of data over the expected background is observed at either of the distributions.

\footnotetext{
$\overline{\text { aincluding true } \gamma \gamma, j} e t+\gamma \rightarrow \gamma_{f a k e} \gamma$ and jet + jet $\rightarrow$ $\gamma_{f a k e} \gamma_{f a k e}$ events

${ }^{\mathrm{b}}$ due to energy mis-measurement in the calorimeter ${ }^{c}$ electromagnetic showers due to cosmic rays and beam halo muons

${ }^{\text {d}}$ Scalar sum of $E_{T}$ 's of all identified objects: photons, electrons, jets, and $\mathbb{F}_{T}$.
}

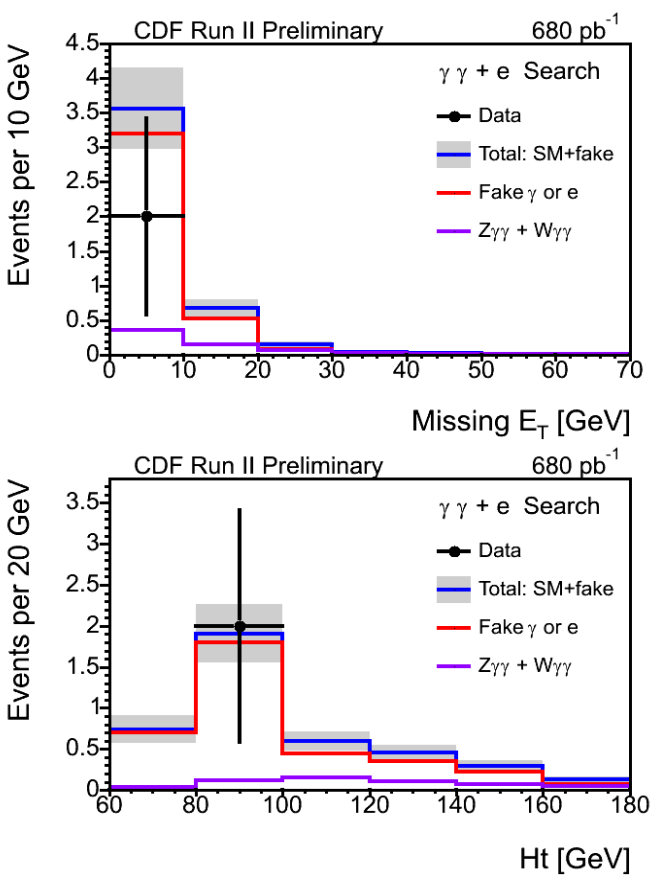

Fig. 3. Distribution of $\mathbb{F}_{T}($ top $)$ and $H_{T}$ (bottom) in $\gamma \gamma+e$ events.

\subsection{The $\gamma \gamma+\gamma$ Channel}

The search for an anomalous production of tri-photon events is based on $\sim 1.2 \mathrm{fb}^{-1}$ of data. All three photons are required to be isolated and have $E_{T}^{\gamma}>13 \mathrm{GeV}$ and $\left|\eta_{\gamma}\right|<1.1$. We observe 4 tri-photon events with the expected background of $2.2 \pm 0.7$ events. There are two known Standard Model contributions to this channel: true tri-photon production; and events with at least one $j e t \rightarrow \gamma_{\text {fake }}$. All of the studied kinematic distributions $\left(E_{T}^{\gamma_{3}}\right.$, $M_{\gamma \gamma}, M_{3 \gamma}, H_{T}$ and $\mathbb{F}_{T}$ ) show good agreement between data and the predicted background.

\subsection{The $\gamma \gamma+e, \mu$ Channel}

CDF has also searched for an anomalous production of $\gamma \gamma$ events $\left(E_{T}^{\gamma}>13 \mathrm{GeV},\left|\eta_{\gamma}\right|<1.1\right)$ in association with an additional electron $\left(E_{T}^{e}>20 \mathrm{GeV},\left|\eta_{e}\right|<2.0\right)$ or a muon $\left(P_{T}^{\mu}>20\right.$ $\left.\mathrm{GeV} / \mathrm{c},\left|\eta_{\mu}\right|<1.0\right)$. This analysis is based on $\sim 680 \mathrm{pb}^{-1}$ of data. Two events are observed 

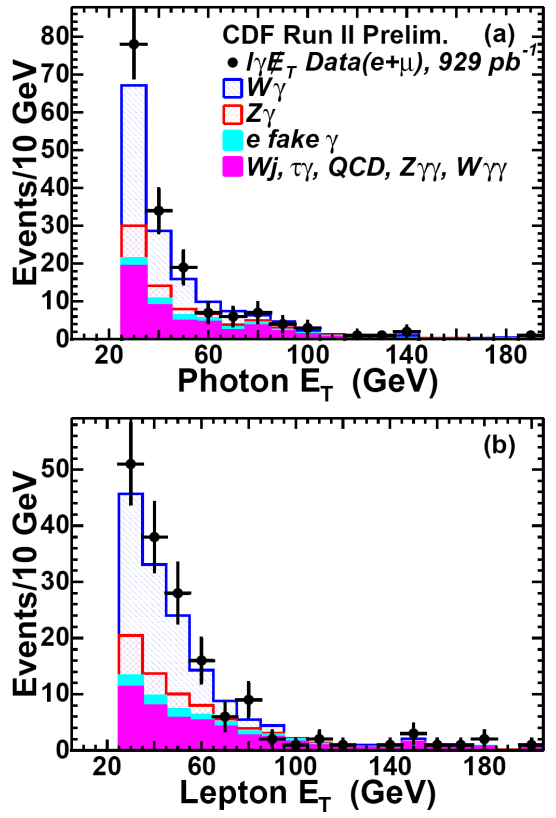

Fig. 4. Distribution of $E_{T}^{\gamma}$ and $E_{T}^{l}$ in $\gamma l+\not_{T}$ events.

in the electron channel which is in agreement with the expected background of $4.49 \pm 0.84$ events. The major source of background for $\gamma \gamma+e$ is events where the electron or one of the photons is fake. No events with an extra muon are found. The expected background for the muon channel is $0.47 \pm 0.12$ events, and it is dominated by the $Z \gamma \gamma$ and $W \gamma \gamma$ production. As a part of this search, we study various kinematic distributions, two of which, namely $\mathbb{F}_{T}$ and $H_{T}$ in $\gamma \gamma+e$ events, are presented at Fig. 3. Data on these distributions agree well with the expected background.

\section{Search for Anomalous Production of $\gamma l+X$ Events}

A search for anomalous inclusive production of events containing a photon and a lepton along with any additional particles is interesting in many ways. Events with photons and leptons are potentially related to the Run I $\gamma \gamma e e \mathbb{F}_{T}$ event ${ }^{1}$. In many theoretical models, new massive particles could undergo
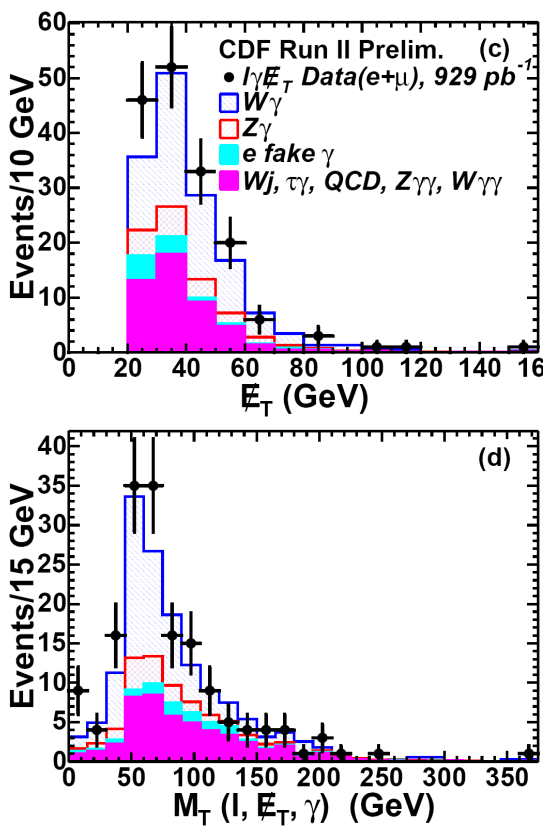

Fig. 5. Distribution of $M_{T}$ and $\not_{T}$ in $\gamma l+\mathbb{F}_{T}$ events.

a cascade decays to Standard Model gauge bosons, one of which could be a photon and the other a $W$ or $Z^{0}$ decaying leptonically. The $\gamma l+X$ search also compliments similar searches in $\gamma \gamma+X, \gamma+j e t+X$, and $\gamma+b+X$ channels. Finally, this new CDF analysis with $\sim 930 \mathrm{pb}^{-1}$ of data will significantly update the results of previous CDF searches ${ }^{3}$ in this channel and shed light on the potential excess in $\gamma l \mathbb{F}_{T}$ channel observed in the Run I analysis ${ }^{3}$. In the current analysis, we require e one isolated central photon and lepton $(e$ or $\mu)$ with $E_{T}^{\gamma, l}>25 \mathrm{GeV}$. Then, the search is split in two modes: the $\gamma l+\not_{T}$ channel, where $\mathbb{E}_{T}>25 \mathrm{GeV}$ and $l=e, \mu$; and the $\gamma l+l$ channel, where $l=e, \mu$. Any additional leptons in the $\gamma l l$ channel are required to pass relaxed requirements ${ }^{3}$ : central $\mu$ and $e$ with $E_{T}>20 \mathrm{GeV}$; and forward $e$ with $E_{T}>15 \mathrm{GeV}$. In the $\gamma l \mathbb{F}_{T}$ channel, we find 163 events corresponding to an expected background of $148.1 \pm 13.0$ events with

$\overline{\mathrm{e}}$ All requirements are identical to those in the Run I search $^{3}$ 


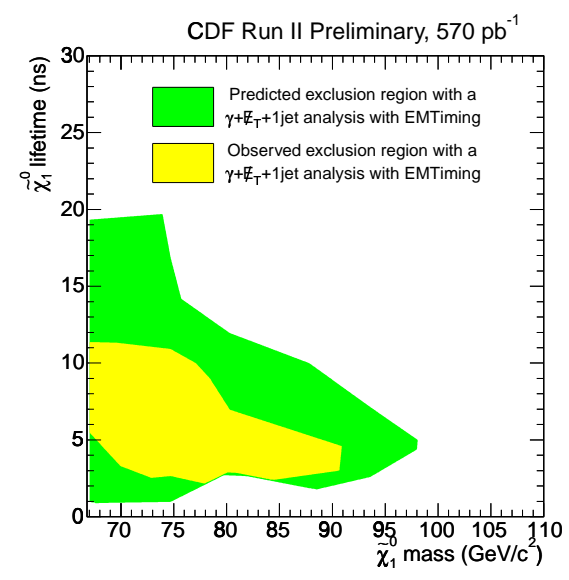

Fig. 6. Exclusion region for $\tilde{\chi}_{1}^{0}$ production.

the dominant contribution from the inclusive $W \gamma$ production. Figs. 4,5 show that data agree well with the predicted background distributions for $E_{T}^{\gamma, l}, M_{T}$ and $\mathscr{F}_{T}$. We also observe $3 \gamma l l \mathbb{F}_{T}$ events with an expected background of $0.6 \pm 0.1$ events. No events with an extra photon are found in the $\gamma l \mathbb{F}_{T}$ channel. In the $\gamma l l$ channel, we observe 74 events which is in agreement with the expected background of $64.9 \pm 7.7$ events, dominated by the inclusive $Z \gamma$ production. No $e \mu \gamma$ events or events with extra leptons and photons are found. Data is consistent with the background predictions on all of the studied kinematic distributions $\left(E_{T}^{l}, E_{T}^{\gamma}, M_{l l}\right.$, and $\left.M_{l l \gamma}\right)$.

\section{Search for Delayed Photons}

Most searches assume that new particles are produced promptly and registered by detectors in time with the other products of the hard collision. However, it is possible that new exotic particles can have large mass, long lifetime, and move with low velocity. These objects can be charged or neutral and decay inside or outside of the detector volume. An example of a theory that offers such a scenario is the GMSB. The model predicts that neutralino, $\tilde{\chi}_{1}^{0}$, has a mass of $\sim 100$ $\mathrm{GeV} / \mathrm{c}^{2}$, lifetime of the order of nanosec- onds and $\sim 100 \%$ branching ratio for $\tilde{\chi}_{1}^{0} \rightarrow \tilde{G} \gamma$ where $\tilde{G}$ is an undetectable gravitino. Therefore, pair production of $\tilde{\chi}_{1}^{0}$ s in association with jets would result in a signature of a delayed photon, large $\mathbb{F}_{T}$, and jets. This search is performed at CDF with $570 \mathrm{pb}^{-1}$ of data. The analysis is optimized to GMSB and requires a photon and a jet with $E_{T}>30$ $\mathrm{GeV}$, and $\not_{T}>50 \mathrm{GeV}$. We observe 10 events where a photon's arrival time relative to prompt particles is $1.5 \mathrm{~ns}<T_{\gamma}<10 \mathrm{~ns}$. This is consistent with $7.6 \pm 1.9$ events of the expected background due to Standard Model $\gamma$ candidates and electromagnetic showers from cosmic ray and beam halo muons that bremsstrahlung in the calorimeter. In the absence of an excess, we set a limit on $\tilde{\chi}_{1}^{0}$ production in the GMSB scenario (Snowmass Slope parameters ${ }^{4}$ ) as a function of its mass and lifetime, see Fig.6.

\section{Summary}

We have presented new results ${ }^{5}$ on two model-independent searches for anomalous production of $\gamma \gamma+X$ and $\gamma l+X$ events. In both analyses, data are consistent with the background expectations. Therefore, the Run I anomalies seen in these channels are not confirmed. We have also presented the results ${ }^{5}$ of a novel search for delayed photons in the context of the GMSB model.

\section{References}

1. F. Abe et al. (CDF Collaboration), Phys. Rev. Lett. 81, 1791 (1998); Phys. Rev. D59, 092002 (1999).

2. V. Abazov et al. (D0 Collaboration), Phys. Rev. Lett. 94, 041801 (2005).

3. D. Acosta et al. (CDF Collaboration), Phys. Rev. Lett. 89, 041802 (2002); Phys. Rev. D71, 032001 (2005); A. Abulencia et al. (CDF Collaboration), Phys. Rev. Lett. 97, 031801 (2006)

4. B.C. Allanach et al., Eur. Phys. J. C25, 113 (2002).

5. See more information at http://wwwcdf.fnal.gov/physics/exotic/exotic.html 